\title{
Immunomodulatory Effects of Vitamin D and Vitamin C to Improve Immunity in COVID-19 Patients
}

\author{
Ahmed Yaqinuddin ${ }^{1}$ Ayesha Rahman Ambia' Raghad A. Alaujan ${ }^{1}$ \\ ${ }^{1}$ Department of Anatomy and Genetics, College of Medicine, \\ Alfaisal University, Riyadh, Saudi Arabia

\begin{abstract}
Address for correspondence Ahmed Yaqinuddin, MD, PhD, FRCP,Department of Anatomy and Genetics, College of Medicine, Alfaisal University, Takhassusi Road, Riyadh 11533, Saudi Arabia (e-mail: ayaqinuddin@alfaisal.edu).
\end{abstract}

J Health Allied Sci NU 2022;12:1-6.

\begin{abstract}
Keywords

- vitamin C

- vitamin D

- COVID-19

- immune response

- chronic diseases

Severe acute respiratory syndrome coronavirus 2 (SARS-COV2) infection causes life-threatening respiratory illness, which has caused significant mortality and morbidity around the globe. Coronavirus disease 2019 (COVID-19) causes mild respiratory illness in most infected individuals; however, in some patients it may progress to sepsis, acute respiratory distress syndrome (ARDS), cytokine release syndrome (CRS), and multiorgan dysfunction (MODS), which results in intensive care unit (ICU) admissions and increased fatalities. Recent evidence shows that most of these comorbidities associated with COVID-19 infection are associated with dysregulation of the host immune response. Vitamins $C$ and $D$ have been shown to regulate immune response by decreasing the proinflammatory cytokine release from immune cells and inducing proliferation of other immune cells to robustly fight infection. This review critically evaluates the current literature on vitamins $C$ and $D$ in modulating an immune response in different diseases and their potential therapeutic effects in preventing complications in COVID-19 infection.
\end{abstract}

\section{Introduction}

Severe acute respiratory syndrome coronavirus 2 (SARS-CoV2) is a zoonotic novel coronavirus originating in China, December 2019, and leading to the most unstoppable infectious disease emergency in the 21st century. With no prior immunity to the disease, most patients develop flu-like respiratory symptoms or remain asymptomatic. The wide variety of symptoms in coronavirus disease 2019 (COVID-19) from fever, dry cough, dyspnea, and dysgeusia to acute respiratory syndrome (ARDS) and cytokine storm ${ }^{1}$ makes it a tricky infectious disease to navigate and manage. So far, there have been multitudes of clinical trials on a range of treatment modalities, targeting specific constituents of the virus or the body's immune system such as vaccines, antivirals, hydroxychloroquine, recombinant soluble angiotensin converting

published online May 12, 2021
DOI https://doi.org/ $10.1055 / \mathrm{s}-0041-1730084$ ISSN 2582-4287 enzyme-2 (ACE2), type 1 interferons, convalescent plasma, corticosteroids, cytokine inhibition by monoclonal antibodies (tocilizumab, interleukin 6 [IL-6] receptor antagonist and anakinra, recombinant interleukin 1 [IL-1] receptor antagonist), intravenous immunoglobulins (IVIG), and lastly inhibition of Janus kinases (JAK). ${ }^{2}$

Even though most clinical presentations are mild, some patients may progress to sepsis, ARDS, cytokine release syndrome (CRS), or multiorgan dysfunction (MODS), leading to intensive care unit (ICU) monitoring and control..$^{1,3}$ There is no specific approved treatment to prevent these comorbidities; vaccines are still undergoing trials. The supportive management includes oxygen maintenance, intravenous fluids, and symptomatic management. Other treatment modalities may include corticosteroids, anticoagulants, tocilizumab, a humanized monoclonal antibody against IL-6 receptor, 
or anakinra, a recombinant monoclonal body against IL-1 receptors. $^{3}$

Most of the case fatalities in COVID-19 patients are seen in the severe presentation. With the onset of cytokine storm being the main etiology of pulmonary destruction also seen in the severe acute respiratory syndrome (SARS) epidemic, it should only make sense that we would propose mechanisms and modalities that target the hallmark of the disease, that is, CRS. There are treatments that target the viral life cycle molecules and treatments that focus on the inflammatory response. In this article we would like to propose actionable steps through the use of accessible, economic, and functional compounds for immunomodulation in COVID-19; we hypothesize that vitamins $\mathrm{D}$ and $\mathrm{C}$ decrease the proinflammatory cytokine release in some components of the immune system and induce proliferation of other immune cells to robustly fight infection and not deplete immune and energy reserves in the host. Vitamin D and vitamin C are immunomodulating agents that have been used for their disease-modifying properties for decades.

\section{COVID-19 and Immunology}

SARS-CoV2 is an encapsulated positive-sense, singlestranded RNA (+sSRNA) virus from the Coronaviridae family. Coronaviruses are spherical with homotrimer projections on the surface called spike (S) proteins. The $S$ protein facilitates adhesion to host cells and binding to transmembrane ACE2 receptor for cell entry. Around 80\% of COVID-19 infections are either asymptomatic or mild illness; the rest are critically ill or requiring intensive care in the ICU. The incidence of SARS-CoV2 is higher in men; this is explained by the fact that androgens from the prostate gland lead to expression of TMPRSS2, which is used as a cofactor to ACE2. The most important cytokine mediating the "cytokine storm" picture in COVID-19 is IL-6, which helps in the release of acute phase reactants, B-cell differentiation, and thermoregulation. COVID-19 has also shown higher coagulation levels of coagulation, with higher levels of thromboembolism and "microclots" in the circulation.,

The innate immune system encountering the SARSCoV2 virus is hypothesized to recognize the ssRNA virus through pattern recognition receptors (PRR) such as cytosolic RIG-I like receptors (RLRs) and toll-like receptors (TLRs). This is followed by cytokine release of antiviral cytokines such as interferons and proinflammatory cytokines such as tumor necrosis factor $\alpha$ (TNF- $\alpha)$, IL-1, IL-6, and IL-18. The host cells clear out viral infections primarily through type 1 interferon clearance. Studies have shown that dysregulation of myeloid cells such as monocytes, macrophages, and dendritic cells leads to ARDS and CRS.

Flow cytometry of peripheral mononuclear cells has shown inflammatory monocytes secreting granulocyte-macrophage colony-stimulatory factor (GM-CSF). IL-6, IL-1, and IFNI/III secreted by the pulmonary epithelium recruit these inflammatory monocytes, neutrophils, and lymphocytes. Inflammatory monocytes, in turn, secrete IL- 6 and TNF- $\alpha$, leading to severe hyperinflammation, and consequently CRS and ARDS. T-lymphocytes, releasing cytokines and mediating cytotoxic effects, and B lymphocytes, secreting antibodies, are also implicated in the defense against COVID-19. ${ }^{1,2}$

\section{Natural Roles of Vitamins D and C in Humans}

\section{Vitamin D}

Vitamin D is an essential fat-soluble vitamin that is obtained from the diet or through ultraviolet- mediated biosynthesis giving rise to its active form 1, 25-di-hydroxycholecalciferol $\left(1,25(\mathrm{OH})_{2} \quad \mathrm{D}_{3}\right)$ or vitamin $\mathrm{D}_{3}$ (calcitriol). Cholecalciferol $\left(D_{3}\right)$ is the primary source of vitamin $D$; ergocalciferol $\left(D_{2}\right)$ is another source. ${ }^{4}$ Vitamin $D_{3}$ is an essential endocrine compound for calcium and phosphorus metabolism and homeostasis, involving the bone, gut, kidneys, and parathyroid glands. ${ }^{5}$ Vitamin $\mathrm{D}$ has been shown to improve immune response against bacteria, viruses, fungi, and parasites. Vitamin $\mathrm{D}_{3}$ binds to a vitamin $\mathrm{D}$ receptor (VDR), which interacts with the retinoic acid receptor (RXR) to form a heterodimer and activates the VDR response elements (VDRE). This $\mathrm{D}_{3} / \mathrm{VDR} / \mathrm{RXR} / \mathrm{VDRE}$ complex is responsible for regulating 900 genes. The vitamin $\mathrm{D}_{3}$-activating enzyme has been expressed in many other tissues such as bone, kidneys, intestine, pancreas, platelets, and prostate. ${ }^{6}$

\section{Vitamin C}

Vitamin C is an important micronutrient and antioxidant involved in many redox reactions. Discovered in the 1920s by Nobel laureate Albert Szent-Györgyi from Hungary as an essential vitamin for scurvy prevention, vitamin $\mathrm{C}$ also has significant immunomodulatory, antimicrobial, antiparasitic, antiviral, and antioxidant properties. ${ }^{7}$ The natural sources of vitamin $\mathrm{C}$ or ascorbic acid are citrus fruits, mangos, strawberries, papayas, tomatoes, among others. ${ }^{7}$ Vitamin $C$ is an essential water-soluble vitamin in human beings since they cannot synthesize it in their bodies due to a lack of L-glucono- $\gamma$-lactone oxidase. Human beings need a minimum of $10 \mathrm{mg}$ daily dose of vitamin C to ward off scurvy, although the daily requirement from diet needs 100 to $200 \mathrm{mg}$ of vitamin $C$ per day for human beings. ${ }^{8}$ Vitamin $C$ also has a partial role in metabolism including energy transformation, collagen biosynthesis and repair, adrenal steroid and catecholamine production, and iron absorption. ${ }^{7,9-11}$ Vitamin $\mathrm{C}$ is also an important cofactor in collagen synthesis, stabilizing the collagen tertiary structure by enabling reactions that require prolyl and lysyl hydroxylase. ${ }^{12,13}$

\section{Molecular Mechanism and Immunomodulating Effects of Vitamin D and Vitamin C}

\section{Vitamin D}

Vitamin $\mathrm{D}_{3}$ is produced in the skin via ultraviolet $\mathrm{B}$ (UVB) radiation, leading to the formation of 7-dehydrocholesterol in the skin, which is followed by a thermal 
reaction. ${ }^{14}$ The liver converts vitamin $\mathrm{D}_{3}$ or oral vitamin $\mathrm{D}$ to 25(OH)D using 25-hydroxylase, and then to the hormonal metabolite $1,25(\mathrm{OH}) 2 \mathrm{D}$ (calcitriol) in the kidneys or other organs through 1- $\alpha$ hydroxylase, as needed. The majority of vitamin $\mathrm{D}$ effects result from calcitriol entering the nuclear vitamin D receptor, which is a DNA-binding protein that interacts directly with regulatory sequences near target genes, resulting in recruitment of chromatin active complexes, participating epigenetically and genetically in altering the transcriptional output. ${ }^{15}$ The expression of the nuclear vitamin D receptors (VDR) in immune cells, such as macrophages, T cells, B cells, NK cells, and dendritic cells (DC), leads to histone acetylation and epigenetic transformation. ${ }^{16}$ In various studies, vitamin D was considered to reduce the risk of viral infections. One recent review about vitamin D's role in reducing the risk of the common cold has grouped these mechanisms into three categories-physical barrier, natural cellular immunity, and adaptive immunity. ${ }^{17}$ Besides, vitamin D helps maintain tight, gap, and adherent junctions (e.g., via E-cadherin). ${ }^{18}$ It is proven by several studies that viruses increase infection by disrupting the junction integrity. ${ }^{19-21}$

Vitamin D is implicated in the biosynthesis of antimicrobial peptides such as cathelicidin (LL-37) and $\beta 2$ defensins, activated B-cells and T-cells, and also promotes proliferation of T-regulatory (Treg) cells. ${ }^{16,22-24}$ Cathelicidins act as direct antimicrobial molecules against Gram-positive (GP) and Gram-negative bacteria (GN), enveloped and nonenveloped viruses, and fungi. ${ }^{25}$ Furthermore, these antimicrobial peptides disturb the cellular membrane of invading pathogens and can neutralize the biological activities of endotoxins resulting in their death. ${ }^{26}$ Other laboratory studies have shown more critical important functions on the antimicrobial peptides including LL-37 reducing influenza A virus replication in a mouse model, ${ }^{27}$ and $1,25(\mathrm{OH}) 2 \mathrm{D}$ reducing the replication of rotavirus both in vitro and in vivo. ${ }^{28}$ The innate immune system generates both proinflammatory and anti-inflammatory cytokines in response to viral and bacterial infections, as observed in COVID-19 patients; and vitamin D has proven to boost the immune system. ${ }^{29}$ Administering vitamin D reduces the expression of proinflammatory Th1 cytokines, such as TNF- $\alpha$, IL-2, and interferon $\gamma$ (IFN- $\gamma$ ), ${ }^{30}$ and increases the expression of anti-inflammatory cytokines by macrophages, thus, signifying its immunomodulatory roles in adaptive immunity. ${ }^{31,32}$ Also, $1,25(\mathrm{OH}) 2 \mathrm{D}_{3}$ promotes T helper type 2 (Th2) cells to produce cytokines that helps to enhance the indirect Th1 cells suppression along with actions mediated by a multitude of cell types. ${ }^{31}$ Additionally, it promotes T regulatory cell induction, thus inhibiting inflammatory processes. ${ }^{33}$ Therefore, vitamin D immunomodulation includes attenuation of the Th1 cells and activation of the Th2 response. It induces synthesis and secretion of anti-inflammatory cytokines such as (IL-4 and IL-10) and inhibits proinflammatory cytokines (IL-1, TNF- $\alpha$, IFN- $\gamma){ }^{6}$

\section{Vitamin C}

Vitamin C reduces the risk of infections as it has antimicrobial properties and immunomodulatory functions, especially in high concentrations. ${ }^{7}$ Vitamin $\mathrm{C}$ can inhibit the activation of nuclear factor kappa-B (NFKB), a primary proinflammatory transcription factor with a crucial role in overall immunity, including cytokines genetic regulations, chemokines, inflammatory mediators, adhesion molecules, and apoptosis inhibitors. ${ }^{34}$ Additionally, vitamin $\mathrm{C}$ can also inhibit the production of TNF- $\alpha$ and IL- 6 , where the effects appear to be dose dependent. ${ }^{35,36}$ Moreover, vitamin $C$ is proven to reduce the GM-CSF signaling responses, which regulate cytokines redox-signal transduction in host defense cells along with a possible role in controlling inflammatory responses. ${ }^{37}$ Furthermore, some studies have shown that high doses of vitamin $C$ have a role in regulating the proliferation and function of $\mathrm{T}$ cells, B cells, and natural killer (NK) cells $\mathrm{s}^{38,39}$ thus, vitamin $\mathrm{C}$ helps in inhibiting the progression of cytokine storms and improves the host's immunity. Moreover, vitamin C can inhibit oxidative stress, ${ }^{35}$ which is an essential part of the innate immune response to viral respiratory infection (e.g., in COVID-19 as oxidative stress may play a role in its mechanism). ${ }^{40,41}$ Additionally, vitamin C can repair oxidative damage in bronchial epithelium by modulating reactive oxygen species (ROS) generation and expression, therefore preventing ROS-induced lung damage. ${ }^{42,43}$ Vitamin $C$ has a role in the epigenetic and transcriptional enhancement of protein channels that regulate alveolar fluid clearance, leading to enhancement of lung epithelial barrier function, improving ARDS symptoms and respiratory function. ${ }^{42,44}$ Some in vitro studies established the vitamin C antiviral effects by inhibiting replication of herpes simplex virus 1 , poliovirus type 1 , and influenza A virus. ${ }^{45}$ Vitamin $C$ has been reported as an alternative agent against sepsis; therein, it was revealed in some studies that high doses of vitamin C might reduce sepsis-related inflammation and vascular injury. ${ }^{46,47}$ Vitamin C supplementation has confirmed beneficial effects against different types of viral infections; furthermore, studies have established that patients with a viral infection, ${ }^{48}$ sepsis, ${ }^{47}$ sepsis-related ARDS $^{49}$ had reduced ascorbate levels. Vitamin $C$ has shown improved survival in lethal infections of different murine models. A study demonstrated the treatment of Venezuelan encephalitis virus infection in mice with vitamin C (50 mg/kg) exhibited reductions in viral titers, products of lipid peroxidation, and NO content. ${ }^{50}$ Additionally, mice incapable of vitamin $\mathrm{C}$ synthesis ( L-gulono-gamma-lactone oxidase nulls) were infected with influenza; not receiving vitamin C supplementation exhibited more outstanding lung pathology scores despite the same viral titers. ${ }^{51}$ Vitamin C was found to reduce capillary-alveolar damage and mortality dose-dependently (100\% versus $80 \%$ versus $50 \%$ at 0,125 , and $250 \mathrm{mg} / \mathrm{kg} /$ day ) in restraint-stressed mice with H1N1 viral-induced pneumonia. ${ }^{52}$

The immunomodulating effects of vitamins $C$ and $D$ can be summarized in - Table $\mathbf{1 .}^{4-8,16,53,54}$

\section{Immunomodulating Effects of Vitamins D and $C$ in Disease and COVID-19}

Vitamin D has been associated with various autoimmune diseases, cancers, and pregnancy outcomes. Vitamin D has led 
Table 1 The immunomodulating effects of vitamins $C$ and $D$

\begin{tabular}{|l|l|}
\hline Vitamin D & Vitamin C \\
\hline $\begin{array}{l}\text { Antimicrobial effects through } \\
\text { cathelicidin and } \beta 2 \text { defensins }\end{array}$ & $\begin{array}{l}\text { Antioxidant, antimicrobial, } \\
\text { antiviral, and antiparasitic } \\
\text { effects }\end{array}$ \\
\hline $\begin{array}{l}\text { Upregulation of Th2 response and } \\
\text { downregulation of Th1 response }\end{array}$ & $\begin{array}{l}\text { Antiapoptosis of monocytes } \\
\text { and apotosis of neutrophils }\end{array}$ \\
\hline $\begin{array}{l}\text { Induces anti-inflammatory } \\
\text { cytokines release and decreases } \\
\text { proinflammatory cytokines }\end{array}$ & $\begin{array}{l}\text { Synthesis of other antioxi- } \\
\text { dants such as glutathione } \\
\text { and tocopherol (vitamin E) }\end{array}$ \\
\hline $\begin{array}{l}\text { T cell proliferation and differ- } \\
\text { entiation, especially Treg over } \\
\text { cytotoxic T cells }\end{array}$ & $\begin{array}{l}\text { Increased immunoglobulin } \\
\text { secretion }\end{array}$ \\
\hline $\begin{array}{l}\text { Maintaining immune tolerance } \\
\text { by APCs }\end{array}$ & $\begin{array}{l}\text { T cell proliferation and } \\
\text { activation }\end{array}$ \\
\hline Activation of TLRs & Accelerated NK cell growth \\
\hline $\begin{array}{l}\text { NK growth, activation and release } \\
\text { of IL-4 and IFN-y by NK cells }\end{array}$ & $\begin{array}{l}\text { Chemotaxis of neutrophils, } \\
\text { macrophages }\end{array}$ \\
\hline $\begin{array}{l}\text { Antiphagocytic activity and IL-1 } \beta \\
\text { and IL-8 release by PMNs and } \\
\text { macrophages }\end{array}$ & $\begin{array}{l}\text { Enhanced phagocytosis and } \\
\text { inhibition of necrosis }\end{array}$ \\
\hline $\begin{array}{l}\text { Decreased B cell proliferation and } \\
\text { immunoglobulin production }\end{array}$ & $\begin{array}{l}\text { Epigenetic } \\
\text { immunomodulation }\end{array}$ \\
\hline Epigenetic immunomodulation & cells; intererom, inter \\
\hline Abbrevions: aPC
\end{tabular}

Abbreviations: APC, antigen-presenting cells; IFN, interferon; IL, interleukin; NK, natural killer; PMNs, polymorphonuclear cells; Th, T helper; TLR, toll-like receptors; Treg, $\mathrm{T}$ regulatory.

to improved pregnancy outcomes, such as reducing miscarriages, intrauterine growth restriction (IUGR), preeclampsia, and pulmonary embolism. It has also been associated with diseases such as rheumatoid arthritis (RA), systemic lupus erythematosus (SLE), multiple sclerosis (MS), antiphospholipid syndrome, and Hashimoto's thyroiditis. ${ }^{16,53}$

Vitamin D activating enzyme 1- $\alpha$ hydroxylase has shown to be upregulated in antigen-presenting cells (APCs), suggesting that calcitriol synthesis in these cells mediates antimicrobial action. One study showed that an IL-15 administration in macrophages led to more substantial vitamin D influence for antimicrobial activity, than IL-10 and vitamin D coadministration in macrophages. Vitamin D has also shown to be markedly decreased in human immunodeficiency virus (HIV) and influenza virus and has been linked with HIV progression and mortality. In children, vitamin D also reduces the occurrence of upper respiratory tract infection (URTI) and decreases the viral load of hepatitis C. Calcitriol, however, also decreases polymorphonuclear cells (PMNs) activity. ${ }^{6}$

Vitamin D is also an immunomodulatory molecule for the innate immune system, leading to increased chemotaxis, phagocytosis, phagolysosomal fusion, and improved barrier function in innate cells. Cathelicidin and $\beta 2$ defensins are produced through vitamin $\mathrm{D}_{3}$ upregulation of cAMP in macrophages, monocytes, and epithelial cells. It has also been shown to increase cAMP and decrease anti-inflammatory cytokines in adults with sepsis. Such an association was not observed in children, however. Vitamin $\mathrm{D}_{3}$ has also shown upregulation of Tregs in type- 1 diabetes.,
The role of vitamin $D$ in autoimmune diseases has been contentious, however. A positive correlation was seen between inflammatory bowel disease (IBD) and vitamin D deficiency, but no such association was noted with Crohn's disease. Vitamin D was associated with increased cytokines such as IL-10, IL-4, and IL-6 in IBD, showing decreased IFN- $\gamma$ compared with control. Multiple sclerosis (MS) has shown to have increased VDRs and 1- $\alpha$ hydroxylase, suggesting the utilization of the anti-inflammatory effects of vitamin $\mathrm{D}$, namely the upregulation of Tregs and decreased cytokine secretion by CD4+ T cells. On the other hand, VDR polymorphisms have been associated with increased risk of RA, especially the Fok1 and Taq1 polymorphisms, suggesting that vitamin D may be positively associated with RA disease. ${ }^{4}$

The discrepancies in certain autoimmune studies may be due to the mode of treatment given, whether the studies were done on animals or humans, underlying diseases, genetic and lifestyle factors, and the different doses of vitamin $\mathrm{D}_{3}$ used in the study.

The immunomodulating effects of vitamin $\mathrm{C}$ can be attributed to its antioxidant properties-it can donate electrons and itself get oxidized, thus taking part in a redox reaction. ${ }^{54}$ Vitamin $C$ is needed in minimum concentration to maintain the cellular and humoral immune response; in higher concentrations, it also exerts lymphoproliferative effects, induces natural killer cells activity, and chemotactic effects. Vitamin $\mathrm{C}$ has an important antioxidant function in the gut barrier function and antioxidation, as studies record reduced vitamin $C$ concentrations in IBD. Recurrent infections in Chediak-Higashi syndrome or chronic granulomatous disease (CGD) have shown improved chemotaxis with vitamin $\mathrm{C}$ administration, considered in part due to its microtubule stabilization effect. Although vitamin $C$ did not cure the underlying etiology in both diseases, it enhanced immune function and improved the prognosis. ${ }^{1,8,55}$

Vitamin $C$ has also been shown to inhibit Pseudomonas aeruginosa growth in vitro and Staphylococcus aureus growth and even slightly inhibiting biofilm production in methicillin-resistant Staphylococcus aureus. However, in some cases such as pH-neutral mediums, Staphylococcus aureus was not inhibited; in similar conditions in another study, group A hemolytic streptococci growth was inhibited.

Vitamin C weakly inhibited Escherichia coli ATTC 11775 growth but had substantive antiproliferative effects on Escherichia coli 0157:H7; 10-20 mg of vitamin C in microaerobic conditions could inhibit Helicobacter pylori infection in vitro but promoted its growth in aerobic conditions. This signifies the importance of concentration, bacterial strain, and environmental conditions for vitamin $C$ inhibitory effects. ${ }^{7}$

In its oxidized and active form L-dehydroascorbic acid (DHA), vitamin C also exerts its antiviral effects by inhibiting replication of herpes simplex virus type 1 (HSV 1 ), rabies virus, influenza virus type $\mathrm{A}$, and poliovirus type $\mathrm{A}$. DHA was shown to have a stronger antiviral activity with the addition of $\mathrm{Fe}^{3+}$ to the culture medium growing HSV1 than DHA alone, due to the formation of hydroxyl free radicals induced by $\mathrm{Fe}^{3+} \cdot 45$ 
Studies have shown that high levels of vitamin $\mathrm{D}$ can reduce bleomycin-induced pulmonary fibrosis in mouse studies by decreasing IL- $1 \beta$ produced by pulmonary fibroblasts. Many studies have also shown that higher vitamin $\mathrm{D}_{3}$ levels were associated with better prognosis in acute respiratory tract infections (ARTI) via decreasing proinflammatory cytokine release from macrophages and $\mathrm{T}$ lymphocytes. On the other end, vitamin D deficiency is associated with an increased likelihood of developing ARTIs. ${ }^{56}$

A recent meta-analysis confirmed vitamin C effects on patients with severe sepsis and ARDS, resulting in reduced mechanical ventilation and length of ICU. ${ }^{9}$ In concordance to this finding, it was recently confirmed in a randomized clinical trial involving 167 patients with sepsis and ARDS that showed significant improvement in 28-day mortality and shortening of ICU length of stay following high-dose intravenous vitamin $C$ up to $15 \mathrm{~g}$ per day. ${ }^{49}$ It is noteworthy that a growing number of case reports support the use of high doses of vitamin C in the treatment against COVID-19.

\section{Conclusion}

With the evidence presented on the role of vitamins $C$ and $D$ in autoimmune, infectious, immunodeficiency syndromes and malignancies, it is safe to conclude that these vitamins do in fact have various immunomodulating effects that can change the course of a disease, altering its severity, progression, and even mortality in some cases. We propose for clinical trials to test the benefits, disadvantages, mechanism of action of the use of vitamins C and D medically in COVID-19 patients and whether such changes are clinically significant.

Conflict of Interest

None declared.

\section{References}

1 Felsenstein S, Herbert JA, McNamara PS, Hedrich CM.. COVID-19: immunology and treatment options. Clin Immunol 2020;215-108448

2 Vabret N, Britton GJ, Gruber C, et al. Sinai Immunology Review Project. Immunology of COVID-19: current state of the science. Immunity 2020;52(6):910-941

3 Cascella M, Rajnik M, Cuomo A, Dulebohn SC, Di Napoli $\mathrm{R}$, Features, Evaluation, and Treatment of Coronavirus (COVID-19). Treasure Island, FL: StatPearls; 2020

4 Goldsmith JR. Vitamin D as an immunomodulator: risks with deficiencies and benefits of supplementation. Healthcare (Basel) 2015;3(2):219-232

5 Myszka M, Klinger M. The immunomodulatory role of Vitamin D. Postepy Hig Med Dosw 2014;68:865-878

6 Sassi F, Tamone C, D’Amelio P, Vitamin D. Vitamin D: nutrient, hormone, and immunomodulator. Nutrients 2018;10 (11):E1656

7 Mousavi S, Bereswill S, Heimesaat MM. Immunomodulatory and antimicrobial effects of vitamin C. Eur J Microbiol Immunol (Bp) 2019;9(3):73-79

8 Carr AC, Maggini S. Vitamin C and immune function. Nutrients 2017;9(11):E1211

9 Hemilä H, Chalker E, Vitamin C.Vitamin C can shorten the length of stay in the ICU: a meta-analysis. Nutrients 2019;11(4):E708
10 Johnston CS, Beezhold BL, Mostow B, Swan PD. Plasma vitamin $\mathrm{C}$ is inversely related to body mass index and waist circumference but not to plasma adiponectin in nonsmoking adults. J Nutr 2007;137(7):1757-1762

11 Johnston CS, Corte C, Swan PD. Marginal vitamin C status is associated with reduced fat oxidation during submaximal exercise in young adults. Nutr Metab (Lond) 2006;3:35

12 Kivirikko KI, Myllylä R, Pihlajaniemi T. Protein hydroxylation: prolyl 4-hydroxylase, an enzyme with four cosubstrates and a multifunctional subunit. FASEB J 1989;3(5):1609-1617

13 Ströhle A, Wolters M, Hahn A. Micronutrients at the interface between inflammation and infection-ascorbic acid and calciferol: part 1, general overview with a focus on ascorbic acid. Inflamm Allergy Drug Targets 2011;10(1):54-63

14 Holick MF. Vitamin D deficiency. N Engl J Med 2007; 357(3):266-281

15 Pike JW, Christakos S. Biology and mechanisms of action of the Vitamin D hormone. Endocrinol Metab Clin North Am 2017;46(4):815-843

16 Bivona G, Agnello L, Ciaccio M. Vitamin D and immunomodulation: is it time to change the reference values? Ann Clin Lab Sci 2017;47(4):508-510

17 Rondanelli M, Miccono A, Lamburghini S, et al. Self-care for common colds: the pivotal role of vitamin D, vitamin C, zinc, and Echinacea in three main immune interactive clusters (physical barriers, innate and adaptive immunity) involved during an episode of common colds-practical advice on dosages and on the time to take these nutrients/botanicals in order to prevent or treat common colds. Evid Based Complement Alternat Med 2018;2018:5813095

18 Schwalfenberg GK. A review of the critical role of vitamin $\mathrm{D}$ in the functioning of the immune system and the clinical implications of vitamin D deficiency. Mol Nutr Food Res 2011;55(1):96-108

19 Chen Y, Leng K, Lu Y, et al. Epidemiological features and time-series analysis of influenza incidence in urban and rural areas of Shenyang, China, 2010-2018. Epidemiol Infect 2020;148:e29

20 Kast JI, McFarlane AJ, Głobińska A, et al. Respiratory syncytial virus infection influences tight junction integrity. Clin Exp Immunol 2017;190(3):351-359

21 Rossi GA, Fanous H, Colin AA. Viral strategies predisposing to respiratory bacterial superinfections. Pediatr Pulmonol 2020;55(4):1061-1073

22 Adams JS, Ren S, Liu PT, et al. Vitamin D-directed rheostatic regulation of monocyte antibacterial responses. J Immunol 2009;182(7):4289-4295

23 Laaksi I. Vitamin D and respiratory infection in adults. Proc Nutr Soc 2012;71(1):90-97

24 Liu PT, Stenger S, Li H, et al. Toll-like receptor triggering of a vitamin D-mediated human antimicrobial response. Science 2006;311(5768):1770-1773

25 Herr C, Shaykhiev R, Bals R. The role of cathelicidin and defensins in pulmonary inflammatory diseases. Expert Opin Biol Ther 2007;7(9):1449-1461

26 Agier J, Efenberger M, Brzezińska-Błaszczyk E. Cathelicidin impact on inflammatory cells. Cent Eur J Immunol 2015; 40(2):225-235

27 Barlow PG, Svoboda P, Mackellar A, et al. Antiviral activity and increased host defense against influenza infection elicited by the human cathelicidin LL-37. PLoS One 2011;6(10):e25333

28 Zhao Y, Ran Z, Jiang Q et al. Vitamin D alleviates rotavirus infection through a microrna-155-5p mediated regulation of the TBK1/IRF3 signaling pathway in vivo and in vitro. Int J Mol Sci 2019;20(14):E3562

29 Huang C, Wang Y, Li X, et al. Clinical features of patients infected with 2019 novel coronavirus in Wuhan, China. Lancet 2020;395(10223):497-506 
30 Sharifi A, Vahedi H, Nedjat S, Rafiei H, Hosseinzadeh-Attar MJ. Effect of single-dose injection of vitamin D on immune cytokines in ulcerative colitis patients: a randomized placebo-controlled trial. APMIS 2019;127(10):681-687

31 Cantorna MT, Snyder L, Lin YD, Yang L. Vitamin D and 1,25(OH) 2D regulation of T cells. Nutrients 2015;7(4):3011-3021

32 Gombart AF, Pierre A, Maggini S. A review of micronutrients and the immune system-working in harmony to reduce the risk of infection. Nutrients 2020;12(1):E236

33 Jeffery LE, Burke F, Mura M, et al. 1,25-dihydroxyvitamin D3 and IL-2 combine to inhibit T cell production of inflammatory cytokines and promote development of regulatory $\mathrm{T}$ cells expressing CTLA-4 and FoxP3. J Immunol 2009;183(9):5458-5467

34 Sen CK, Packer L. Antioxidant and redox regulation of gene transcription. FASEB J 1996;10(7):709-720

35 Chen Y, Luo G, Yuan J, et al. Vitamin C mitigates oxidative stress and tumor necrosis factor-alpha in severe community-acquired pneumonia and LPS-induced macrophages. Mediators Inflamm 2014;2014:426740

36 Härtel C, Strunk T, Bucsky P, Schultz C. Effects of vitamin C on intracytoplasmic cytokine production in human whole blood monocytes and lymphocytes. Cytokine 2004;27(4-5):101-106

37 Cárcamo JM, Bórquez-Ojeda O, Golde DW. Vitamin C inhibits granulocyte macrophage-colony-stimulating factor-induced signaling pathways. Blood 2002;99(9):3205-3212

38 Maeng HG, Lim H, Jeong YJ, et al. Vitamin C enters mouse T cells as dehydroascorbic acid in vitro and does not recapitulate in vivo vitamin C effects. Immunobiology 2009;214(4):311-320

39 Tanaka M, Muto N, Gohda E, Yamamoto I. Enhancement by ascorbic acid 2-glucoside or repeated additions of ascorbate of mitogen-induced IgM and IgG productions by human peripheral blood lymphocytes. Jpn J Pharmacol 1994;66(4):451-456

40 Delgado-Roche L, Mesta F. Oxidative stress as key player in severe acute respiratory syndrome coronavirus (SARS-CoV) infection. Arch Med Res 2020;51(5):384-387

41 Kratzer E, Tian Y, Sarich N, et al. Oxidative stress contributes to lung injury and barrier dysfunction via microtubule destabilization. Am J Respir Cell Mol Biol 2012;47(5):688-697

42 Fisher BJ, Kraskauskas D, Martin EJ, et al. Mechanisms of attenuation of abdominal sepsis induced acute lung injury by ascorbic acid. Am J Physiol Lung Cell Mol Physiol 2012;303(1):L20-L32

43 Lang JD, McArdle PJ, O'Reilly PJ, Matalon S. Oxidant-antioxidant balance in acute lung injury. Chest 2002;122(6,Suppl):314S-320S
44 Bharara A, Grossman C, Grinnan D, et al. Intravenous Vitamin C administered as adjunctive therapy for recurrent acute respiratory distress syndrome. Case Rep Crit Care 2016;2016:8560871

45 Furuya A, Uozaki M, Yamasaki H, Arakawa T, Arita M, Koyama AH. Antiviral effects of ascorbic and dehydroascorbic acids in vitro. Int J Mol Med 2008;22(4):541-545

46 Brant EB, Angus DC. Is high-dose vitamin C beneficial for patients with sepsis. ? JAMA 2019;322(13):1257-1258

47 Marik PE, Hooper MH. Doctor-your septic patients have scurvy! Crit Care 2018;22(1):23

48 Chen JY, Chang CY, Feng PH, Chu CC, So EC, Hu ML. Plasma vitamin $C$ is lower in postherpetic neuralgia patients and administration of vitamin $\mathrm{C}$ reduces spontaneous pain but not brush-evoked pain. Clin J Pain 2009;25(7):562-569

49 Fowler AA II, Truwit JD, Hite RD, et al. Effect of vitamin C infusion on organ failure and biomarkers of inflammation and vascular injury in patients with sepsis and severe acute respiratory failure: the CITRIS-ALI randomized clinical trial. JAMA 2019;322(13):1261-1270

50 Valero N, Mosquera J, Alcocer S, Bonilla E, Salazar J, Álvarez-Mon M. Melatonin, minocycline and ascorbic acid reduce oxidative stress and viral titers and increase survival rate in experimental Venezuelan equine encephalitis. Brain Res 2015;1622:368-376

51 Li W, Maeda N, Beck MA. Vitamin C deficiency increases the lung pathology of influenza virus-infected gulo-/- mice. J Nutr 2006;136(10):2611-2616

52 Cai Y, Li YF, Tang LP, et al. A new mechanism of vitamin C effects on $A / F M / 1 / 47(H 1 N 1)$ virus-induced pneumonia in restraint-stressed mice. Bio Med Res Int 2015;2015:675149

53 Cyprian F, Lefkou E, Varoudi K, Girardi G. Immunomodulatory effects of vitamin D in pregnancy and beyond. Front Immunol 2019;10:2739

54 Hartel C, Puzik A, Gopel W, Temming P, Bucsky P, Schultz C. Immunomodulatory effect of vitamin $\mathrm{C}$ on intracytoplasmic cytokine production in neonatal cord blood cells. Neonatology 2007;91(1):54-60

55 Buffinton GD, Doe WF. Altered ascorbic acid status in the mucosa from inflammatory bowel disease patients. Free Radic Res 1995;22(2):131-143

56 Panfili FM, Roversi M, D’Argenio P, Rossi P, Cappa M, Fintini D. Possible role of vitamin D in COVID-19 infection in pediatric population. J Endocrinol Invest 2020 